Discrete Comput Geom 29:625-631 (2003)

DOI: $10.1007 / \mathrm{s} 00454-003-0769-8$

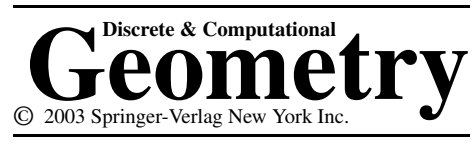

\title{
Perfect Packings of Squares Using the Stack-Pack Strategy
}

\author{
Johan Wästlund \\ Department of Mathematics, Linköping University, \\ S-581 83 Linköping, Sweden \\ jowas@mai.liu.se
}

\begin{abstract}
We consider the problem of packing an infinite set of square tiles into a finite number of rectangular boxes. We introduce a simple packing strategy that we call stackpack. Using this strategy, we prove that if $\frac{1}{2}<t<\frac{2}{3}$, then the squares of side $n^{-t}$, for positive integers $n$, can be packed into some finite collection of square boxes of the same area $\zeta(2 t)$ as the total area of the tiles.
\end{abstract}

\section{Introduction}

We consider an infinite collection of square tiles of side $f(n)$, where $f$ is a function of the positive integers such that

$$
\sum_{n=1}^{\infty} f(n)^{2}<\infty
$$

in other words, the total area of the squares is finite. We can assume that $f$ is weakly decreasing. We may ask whether it is possible to pack the squares into a rectangular box of given dimensions, that is, to place the squares inside the rectangular box so that no two of them have an interior point in common. Such a packing is called perfect if the area of the box is the same as the total area of the squares.

Chalcraft [1] has introduced methods for proving that a given set of squares can be perfectly packed into a rectangular box. He shows that a perfect packing exists provided there is a packing of the first few larger squares satisfying certain inequalities. He then shows that for some exponents $t$ in the interval $\frac{1}{2}<t \leq \frac{3}{5}$, the squares of side $n^{-t}$ can be perfectly packed into a square box of area $\zeta(2 t)$.

We introduce a simpler strategy for packing the squares, and by extending the methods of [1], we prove that perfect packings similar to those of [1] can be constructed for $t<\frac{2}{3}$.

The choice of a single square box is natural but arbitrary. The methods introduced in [1] are more general. An obstacle is that a computation must be made separately for each 
$t$ in order to show that a certain finite number of squares can be packed to begin with. For larger values of $t$ it is no longer possible to pack the squares of side $n^{-t}$ into a square box, since not even the first two squares will fit into the box. One can still conjecture that when $t=1$ for example, a rectangular box with shorter side equal to 1 will do.

To avoid these difficulties, and to treat the various packing problems in a uniform way, we make the following definition:

We say that a collection of square tiles allows a perfect packing if there exists a finite collection of rectangular boxes of the same total area as that of the squares, into which they can be packed.

We will not use this definition in any technical sense. It merely serves to formulate the problem with emphasis on the infinite tail of smaller squares. In particular, when considering the question of the existence of a perfect packing of a given collection of squares, we allow ourselves to discard any finite number of squares, simply by giving each of them their own box.

\section{A Packing Strategy}

We introduce a strategy that we call stack-pack for packing the squares. The stack-pack strategy is based on the following three principles.

1. The Well-Ordering Principle. Pack the squares one at a time, always choosing the largest remaining square.

This implies that if we never get stuck, we are done. If there is no positive integer $n$ for which the $n$th square cannot be packed into the remaining area of the boxes, then the process will lead to a packing of all the squares.

The second principle makes sure that we will always be dealing with a collection of rectangular boxes, and not with the irregular shapes that arise if we start packing squares in a random fashion in a box. This method was introduced in [2].

2. The Divide-and-Conquer Principle. When packing a square into a box, always put it in one of the corners of the box, and then divide the remaining L-shaped region of the box into two smaller rectangular boxes.

Principles 1 and 2 imply that the problem will always stay in its original form, that is, after having packed any finite number of squares, what remains is still the problem of packing the rest of the squares into a finite collection of rectangular boxes. Although the number of boxes grows, the problem does not become conceptually more complicated. As was shown in [1], this may allow for an argument by induction, showing that a perfect packing exists.

We now introduce a third rule, which will put some further constraints on the packing. The benefit of this rule is not so much that it provides a good packing strategy, but that we will be able to analyse it and prove some of its properties. 


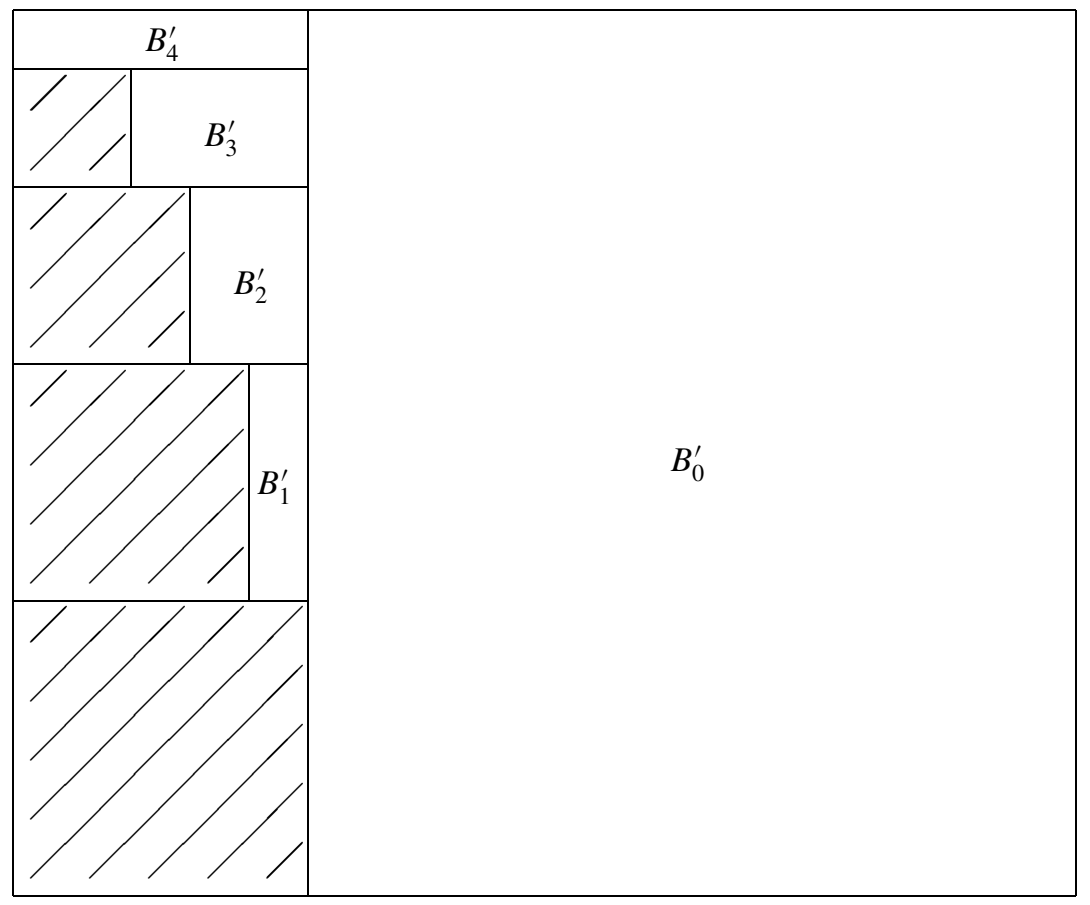

Fig. 1. A round of stack-pack.

3. The Stacking Principle. Choose any box B large enough to contain the next square, and stack as many of the following squares as possible along the shorter side of $B$. Then divide the remaining area of $B$ with a line from the inner corner of the first square parallel to the shorter side of $B$, and further with segments from the corners of the smaller squares perpendicular to, and extending out to, the first line, as in Fig. 1. Then the remaining area of $B$ is divided into boxes $B_{0}^{\prime}, \ldots, B_{k}^{\prime}$, where $k$ is the number of squares that were stacked.

Notice that Principle 3 is consistent with Principles 1 and 2. A sequence of squares stacked according to Principle 3 will be called a round.

\section{Analysis of the Stack-Pack Strategy}

We assume that the stack-pack strategy is used, and let $\mathbf{B}_{n}$ denote the collection of boxes available when $n$ squares have been packed.

If $B$ is a box with sides $x$ and $y$, we let $h(B)=\max (x, y)$ and $w(B)=\min (x, y)$. Moreover, we let $a(B)=h(B) w(B)$ be the area of $B$.

If $\mathbf{B}$ is a collection of boxes, then we let $h(\mathbf{B})=\sum_{B \in \mathbf{B}} h(B)$ and $a(\mathbf{B})=\sum_{B \in \mathbf{B}} a(B)$. Hence $a\left(\mathbf{B}_{n}\right)=\sum_{j=n+1}^{\infty} f(j)^{2}$. 
Since the total area of the boxes is assumed to be equal to the total area of the tiles, the number of squares that can be packed in each round of stack-pack must be finite. This argument further leads to the following inequality:

Theorem 3.1. Suppose that the squares $n+1, \ldots, n+k$ are packed in one round of stack-pack. Then

$$
k^{2} \cdot f(n+k)^{2} \leq \sum_{j=n+1}^{\infty} f(j)^{2}
$$

Proof. Since we assumed that $f$ is weakly decreasing, we have $w(B) \geq k f(n+k)$. Now $w(B)^{2} \leq a(B)$, which is at most the area of the remaining squares.

If we put $f(n)=n^{-t}$, for some $t>\frac{1}{2}$, then (1) implies that for large $n, k$ is at most of order $\sqrt{n}$ :

Theorem 3.2. If $t<\frac{1}{2}$, and $f(n)=n^{-t}$, then under the conditions of Theorem 3.1, $k=O(\sqrt{n})$ as $n \rightarrow \infty$.

Proof. By an integral estimate,

$$
a\left(\mathbf{B}_{n}\right) \leq \int_{n}^{\infty} x^{-2 t} d x=\frac{n^{1-2 t}}{2 t-1}
$$

Hence by (1),

$$
k^{2}(n+k)^{-2 t} \leq \frac{n^{1-2 t}}{2 t-1} .
$$

This can be rearranged as

$$
\frac{k}{\sqrt{n}} \leq \frac{(1+k / n)^{t}}{\sqrt{2 t-1}} .
$$

Here the right-hand side is bounded as $n \rightarrow \infty$, provided that $k / n$ is. Since Theorem 3.1 holds even if $k$ is smaller than the total number of squares packed in the round, we can always choose $k \leq n$, say. This implies that the right-hand side of (2) is bounded by a constant that depends only on $t$ and not on $n$.

As was pointed out by an anonymous referee, (2) itself shows that if $t<1$, then $k=o(n)$ as $n \rightarrow \infty$, and this is all we need for the proof of Theorem 5.1.

There is no guarantee that, in a specific case, we will not run into a dead end using stack-pack. Hence there could be only a finite number of values of $n$ to consider. In such a case it seems meaningless to let $n$ tend to infinity. However, Theorem 3.2 gives a bound on $k$ which is independent of the choices involved in the packing, and this bound, in turn, is of order $\sqrt{n}$ as $n \rightarrow \infty$. 


\section{A Criterion}

We introduce a criterion which is sufficient for the next square to fit into some box.

Theorem 4.1. If $\mathbf{B}$ is a collection of boxes of total area $a(\mathbf{B})$, and

$$
h(\mathbf{B}) \leq \frac{a(\mathbf{B})}{s},
$$

then a square of side $s$ will fit into some box in $\mathbf{B}$.

Proof. A square of side $s$ will fit into some box $B$ in $\mathbf{B}$ if and only if

$$
s \leq \max _{B \in \mathbf{B}}(w(B))
$$

If (3) holds, then

$$
s \leq \frac{a(\mathbf{B})}{h(\mathbf{B})}=\frac{\sum h(B) w(B)}{\sum h(B)} \leq \frac{\max (w(B)) \sum h(B)}{\sum h(B)}=\max (w(B)),
$$

where the sums and maxima are taken over all $B \in \mathbf{B}$.

Next we look at how the stack-pack strategy affects the collection of boxes available. The next theorem gives a bound on the increase in $h(\mathbf{B})$ in one round of stack-pack.

Theorem 4.2. If $n$ squares have been packed, and $k$ more squares are packed in the next round of stack-pack, then

$$
h\left(\mathbf{B}_{n+k}\right)-h\left(\mathbf{B}_{n}\right) \leq k \cdot f(n+1) .
$$

Proof. See Fig. 1 . Let $B$ be the box used in this round, and let $B_{0}^{\prime}, \ldots, B_{k}^{\prime}$ be the $k+1$ new boxes created in this round. We have $h\left(B_{0}^{\prime}\right) \leq h(B)$, and $h\left(B_{i}^{\prime}\right) \leq f(n+1)$ for $1 \leq i \leq k . B_{1}^{\prime}, \ldots, B_{k}^{\prime}$ contributes by at most $f(n+1)$.

\section{Perfect Packings}

We now fix $f(n)=n^{-t}$. Theorem 4.2 gives a bound on the increase in $h(\mathbf{B})$ during one round of stack-pack, and Theorem 4.1 shows that a bound on $h(\mathbf{B})$ may prove that yet another square can be packed. A natural thing to do at this point would be to assume, as an induction hypothesis, that inequality (3) holds, and then to show that provided $n$ is large enough, the inequality still holds after another round of the packing. This would involve making an integral estimate of $a\left(\mathbf{B}_{n}\right)$, introducing an error term. To simplify the calculations, we make the integral estimate first, and then discard the error term when formulating the induction hypothesis.

We have

$$
a\left(\mathbf{B}_{n}\right) \geq \int_{n+1}^{\infty} x^{-2 t} d x=\frac{(n+1)^{1-2 t}}{2 t-1}
$$


Hence

$$
\frac{a\left(\mathbf{B}_{n}\right)}{f(n+1)} \geq \frac{(n+1)^{1-t}}{2 t-1} .
$$

We take as our induction hypothesis that

$$
h\left(\mathbf{B}_{n}\right) \leq \frac{(n+1)^{1-t}}{2 t-1} .
$$

As long as the induction hypothesis is satisfied, we have

$$
h\left(\mathbf{B}_{n}\right) \leq \frac{a\left(\mathbf{B}_{n}\right)}{f(n+1)},
$$

and, by Theorem 4.1, it is possible to pack another square into some box.

Assume that at some stage of the packing, we have

$$
h\left(\mathbf{B}_{n}\right) \leq \frac{(n+1)^{1-t}}{2 t-1},
$$

but that after the next round of stack-pack,

$$
h\left(\mathbf{B}_{n+k}\right)>\frac{(n+k+1)^{1-t}}{2 t-1} .
$$

We show that if $t$ is suitably chosen, and $n$ is large enough, this leads to a contradiction.

By Theorem $3.2 k / n$ tends to zero as $n \rightarrow \infty$. We now show that if $t<\frac{2}{3}$, then (4) and (5) imply that $k / n$ is bounded from below by a constant depending only on $t$ and not on $n$. By Theorem 4.2 we have

$$
\frac{(n+k+1)^{1-t}-(n+1)^{1-t}}{2 t-1}<h\left(\mathbf{B}_{n+k}\right)-h\left(\mathbf{B}_{n}\right) \leq k \cdot(n+1)^{-t} .
$$

We multiply by $2 t-1$ and arrange for every occurrence of $k$ to be divided by $n+1$ :

$$
(n+1)^{1-t}\left(\left(1+\frac{k}{n+1}\right)^{1-t}-1\right)<(2 t-1) \frac{k}{n+1}(n+1)^{1-t} .
$$

We now divide by $(n+1)^{1-t}$ and add 1 :

$$
\left(1+\frac{k}{n+1}\right)^{1-t}<1+(2 t-1) \frac{k}{n+1} .
$$

As $k /(n+1) \rightarrow 0$, the left-hand side is

$$
1+(1-t) \frac{k}{n+1}+O\left(\left(\frac{k}{n+1}\right)^{2}\right) .
$$

Hence if $1-t>2 t-1$, in other words, if $t<\frac{2}{3}$, then (6) implies that $k /(n+1)$ is bounded away from zero, contradicting Theorem 3.2. 
Theorem 5.1. If $\frac{1}{2}<t<\frac{2}{3}$, then the squares of side $n^{-t}$ allow a perfect packing into a set of square boxes.

Proof. We choose $n$ large enough that inequalities (2) and (6) are incompatible. We discard the first $n$ squares by giving each of them a separate box, and start with $\mathbf{B}_{n}$ equal to a single square box of area

$$
\sum_{j=n+1}^{\infty} f(j)^{2}
$$

Since

$$
h\left(\mathbf{B}_{n}\right)=a\left(\mathbf{B}_{n}\right)^{1 / 2} \leq\left(\int_{n}^{\infty} x^{-2 t} d x\right)^{1 / 2}=\frac{n^{1 / 2-t}}{(2 t-1)^{1 / 2}} \leq \frac{(n+1)^{1-t}}{2 t-1},
$$

whenever $n \geq 2 t-1$, the induction hypothesis is satisfied. Therefore the stack-pack strategy will lead to a perfect packing.

\section{References}

1. A. Chalcraft, Perfect square packings, J. Combin. Theory Ser. A 92 (2000), 158-172.

2. M. M. Paulhus, An algorithm for packing squares, J. Combin. Theory Ser. A 82 (1998), 147-157.

Received January 16, 2002, and in revised form December 15, 2002. Online publication April 22, 2003. 